\title{
A Note on the Observability of Temporal Boolean Control Network
}

\author{
Wenping Shi, ${ }^{1}$ Bo Wu, ${ }^{1,2}$ and Jing $\mathrm{Han}^{3}$ \\ ${ }^{1}$ College of Mathematics, Physics and Information Engineering, Zhejiang Normal University, Jinhua 321004, China \\ ${ }^{2}$ Academic Affairs Division, Zhejiang Normal University, Jinhua 321004, China \\ ${ }^{3}$ Department of Mathematics, Tongji University, Shanghai 200092, China
}

Correspondence should be addressed to Bo Wu; wubo_502@163.com

Received 2 December 2012; Accepted 24 February 2013

Academic Editor: Qi Luo

Copyright (C) 2013 Wenping Shi et al. This is an open access article distributed under the Creative Commons Attribution License, which permits unrestricted use, distribution, and reproduction in any medium, provided the original work is properly cited.

\begin{abstract}
Temporal Boolean network is a generalization of the Boolean network model that takes into account the time series nature of the data and tries to incorporate into the model the possible existence of delayed regulatory interactions among genes. This paper investigates the observability problem of temporal Boolean control networks. Using the semi tensor product of matrices, the temporal Boolean networks can be converted into discrete time linear dynamic systems with time delays. Then, necessary and sufficient conditions on the observability via two kinds of inputs are obtained. An example is given to illustrate the effectiveness of the obtained results.
\end{abstract}

\section{Introduction}

Boolean network (BN) is the simplest logical dynamic system. It was proposed by Kauffman for modeling complex and nonlinear biological systems; see [1-3]. Since then, it has been a powerful tool in describing, analyzing, and simulating the cell networks. In this model, gene state is quantized to only two levels: true and false. Then, the state of each gene is determined by the states of its neighborhood genes, using logical rules.

The control of BN is a challenging problem. So far, there are only few results on it because of the shortage of systematic tools to deal with logical dynamic systems; see $[4,5]$. Recently, a new matrix product, which was called the semitensor product (STP) [4], was provided to convert a logical function into an algebraic function, and the logical dynamics of BNs could be converted into standard discrete-time dynamics. Based on this, a new technique has been developed for analyzing and synthesizing Boolean (control) networks (BCNs); see [4, 69]. Furthermore, [10] have presented some simple criteria to judge the controllability with respect to input-state incidence matrices of BCNs. A Mayer-type optimal control problem for BCNs with multi-input and single input has been studied in $[11,12]$.
Systematic analysis of biological systems is an important topic in systems biology, and the observability is a structural property of systems. There have been many results on the controllability and observability of dynamic systems; see [13-18]. When it comes to the observability problem of BNs, Cheng and Qi have obtained necessary and sufficient conditions for the observability of BCNs in [8]. However, simple Boolean method cannot be used to study the kinetic properties of networks because it does not have time components, and time delay behaviors happen frequently in biological and physiological systems. In [19], the observability problem for a class of Boolean control systems with time delay is investigated.

It is well known that time delay phenomenon is very common in the real world $[20,21]$ and very important in analysis and control for dynamic systems. Since many experiments involve obtaining gene expression data by monitoring the expression of genes involved in some biological process (e.g., neural development) over a period of time, the resulting data is in the form of a time series [22]. It is interesting to understand how the expression of a gene at some stage in the process is influenced by the expression levels of other genes during the stages of the process preceding it. Temporal Boolean networks (TBNs) are developed to help model the 
temporal dependencies that span several time steps and model regulatory delays, which may come about due to missing intermediary genes and spatial or biochemical delays between transcription and regulation; see [23-25].

It should be noticed that TBCN is similar with higherorder BCN from Chapter 5 of [26] in which the higher-order $\mathrm{BCN}$ can be rewritten by a BCN by using the first algebraic form of the network. Hence, the observability analysis for higher-order BCNs can be obtained from [26]. However, if the first algebraic form is used, the dimension of network transition matrix depending on the number of logical variables will be much larger which would make computation cost much higher [27]. Motivated by the above analysis, the purpose of this paper is to use STP developed in $[4,6-9,28]$ to analyze the observability problem of TBCN without changing it into $\mathrm{BCN}$, which generalizes the $\mathrm{BN}$ model to cope with dependencies that span over more than one unit of time.

The rest of this paper is organized as follows. Section 2 provides a brief review for the STP of matrices and the matrix expression of logical function. In Section 3, we convert TBCN into discrete time delay systems. In Section 4, necessary and sufficient conditions for the observability of the temporal $\mathrm{BCN}$ are obtained. An example is given to illustrate the efficiency of the proposed results in Section 5. Finally, a brief conclusion is presented.

\section{Preliminaries}

For simplicity, we first give some notations as in [4]. Denote $M_{m \times n}$ as the set of all $m \times n$ matrices. The delta set $\Delta_{k}:=$ $\left\{\delta_{k}^{i} \mid i=1,2, \ldots, k\right\}$, where $\delta_{k}^{i}$ is the $i$ th column of identity matrix $I_{k}$ with degree $k$. A matrix $A \in M_{m \times n}$ is called a logical matrix if the columns set of $A$, denoted by $\operatorname{Col}(A)$, satisfies $\operatorname{Col}(A) \subset \Delta_{m}$. The set of all $m \times n$ logical matrices is denoted by $\mathscr{L}_{m \times n}$. Assuming $A=\left[\delta_{m}^{i_{1}}, \delta_{m}^{i_{2}}, \ldots, \delta_{m}^{i_{n}}\right] \in \mathscr{L}_{m \times n}$, we denote it as $A=\delta_{m}\left[i_{1}, i_{2}, \ldots, i_{n}\right]$.

We recall the concept of STP. Let $X$ be a row vector of dimension $n p$ and $Y$ a column vector of dimension $p$. Then, we split $X$ into equal-sized blocks as $X^{1}, \ldots, X^{p}$, which are $1 \times p$ rows. Define the STP, denoted by $\ltimes$, as

$$
\begin{gathered}
X \ltimes Y=\sum_{i=1}^{p} X^{i} y_{i} \in R^{n}, \\
Y^{T} \ltimes X^{T}=\sum_{i=1}^{p} y_{i}\left(X^{i}\right)^{T} \in R^{n} .
\end{gathered}
$$

In this paper, “ $\ltimes$ " is omitted, and throughout this paper the matrix product is assumed to be the semi-tensor product as in [9].

The swap matrix $W_{[m, n]}$ is an $m n \times m n$ matrix. Label its columns by $(11,12, \ldots, 1 n, \ldots, m 1, m 2, \ldots, m n)$ and its rows by $(11,21, \ldots, m 1, \ldots, 1 n, 2 n, \ldots, m n)$. Then, its element in the position $((I, J),(i, j))$ is assigned as

$$
w_{(I, J),(i, j)}=\delta_{i, j}^{I, J}= \begin{cases}1, & I=i, J=j \\ 0, & \text { otherwise. }\end{cases}
$$

When $m=n$, we briefly denote $W_{[n]}=W_{[m, n]}$. Furthermore, for $X \in \mathbb{R}^{m}$ and $Y \in \mathbb{R}^{n}, W_{[m, n]} \ltimes X \ltimes Y=Y \ltimes X$ and $W_{[n, m]} \ltimes$ $Y \ltimes X=X \ltimes Y$.

A logical domain, denoted by $\mathscr{D}$, is defined as $\mathscr{D}:=\{T=$ $1, F=0\}$. To use matrix expression, we identify each element in $\mathscr{D}$ with a vector as $T \sim \delta_{2}^{1}$ and $F \sim \delta_{2}^{2}$ and denote $\Delta:=$ $\Delta_{2}=\left\{\delta_{2}^{1}, \delta_{2}^{2}\right\}$. Using STP of matrices, a logical function with $n$ arguments $L: \mathscr{D}^{n} \rightarrow \mathscr{D}$ can be expressed in the algebraic form as follows.

Lemma 1 (see [9]). Any logical function $L\left(A_{1}, \ldots, A_{n}\right)$ with logical arguments $A_{1}, \ldots, A_{n} \in \Delta$ can be expressed in a multilinear form as

$$
L\left(A_{1}, \ldots, A_{n}\right)=M_{L} A_{1} \cdots A_{n}
$$

where $M_{L} \in \mathscr{L}_{2 \times 2^{n}}$ is unique which is called the structure matrix of $L$.

Lemma 2 (see [9]). Assume that $P_{k}=A_{1} \cdots A_{k}$ with logical arguments $A_{1}, \ldots, A_{k} \in \Delta$, then

$$
P_{k}^{2}=\Phi_{k} P_{k}
$$

where $\Phi_{k}=\prod_{i=1}^{k} I_{2^{i-1}} \otimes\left[\left(I_{2} \otimes W_{\left[2,2^{k-i}\right]}\right) M_{r}\right], M_{r}=\delta_{4}[1,4]$.

\section{Algebraic Form of Temporal Boolean Networks}

We consider the temporal Boolean network [25] of a set of nodes $A_{1}, \ldots, A_{n} \in \Delta$ as follows:

$$
\begin{aligned}
& A_{i}(t+1) \\
& =f_{i}\left(A_{1}(t), \ldots, A_{n}(t), A_{1}(t-1), \ldots, A_{n}(t-1), \ldots,\right. \\
& \left.\quad A_{1}(t-\tau), \ldots, A_{n}(t-\tau)\right), \quad i=1,2, \ldots, n,
\end{aligned}
$$

where $f_{i}, i=1,2, \ldots, n$ are logical functions, $t=0,1,2, \ldots$, and $\tau$ is a positive integer delay.

Using Lemma 1, for each logical function $f_{i}, i=$ $1,2, \ldots, n$, we can find its structure matrix $M_{i}$. Let $x(t)=$ $\ltimes_{i=1}^{n} A_{i}(t)$. Then, the system (5) can be converted into an algebraic form as

$$
\begin{aligned}
A_{i}(t+1) & =M_{i} \ltimes_{j=1}^{n} A_{j}(t) \cdots \ltimes_{j=1}^{n} A_{j}(t-\tau) \\
& =M_{i} x(t) \cdots x(t-\tau), \quad i=1, \ldots, n .
\end{aligned}
$$


From Lemma 2, multiplying all systems in (6) together yields

$$
\begin{aligned}
x(t+1)= & \ltimes_{i=1}^{n} A_{i}(t+1) \\
= & \ltimes_{i=1}^{n}\left[M_{i} x(t) \cdots x(t-\tau)\right] \\
= & M_{1}\left[\left(I_{2^{n(\tau+1)}} \otimes M_{2}\right) \Phi_{n(\tau+1)}\right] x(t) \cdots \\
& \times x(t-\tau) M_{3} \cdots M_{n} x(t) \cdots x(t-\tau) \\
= & M_{1}\left[\ltimes_{i=2}^{3} I_{2^{n(\tau+1)}} \otimes M_{i} \Phi_{n(\tau+1)}\right] x(t) \cdots \\
& \times x(t-\tau) M_{4} \cdots M_{n} x(t) \cdots x(t-\tau) \\
= & \cdots \\
= & M_{1}\left[\ltimes_{i=2}^{n} I_{2^{n(\tau+1)}} \otimes M_{i} \Phi_{n(\tau+1)}\right] x(t) \cdots x(t-\tau) .
\end{aligned}
$$

Denote $L_{0}:=M_{1}\left[\ltimes_{i=2}^{n} I_{n(\tau+1)} \otimes M_{i} \Phi_{n(\tau+1)}\right]$. Then (7) can be expressed as

$$
x(t+1)=L_{0} x(t) \cdots x(t-\tau),
$$

and $L_{0}$ is called the network transition matrix of (5).

Next, we consider temporal Boolean control network with outputs as follows:

$$
\begin{aligned}
& A_{i}(t+1) \\
& =f_{i}\left(u_{1}(t), \ldots u_{m}(t), A_{1}(t), \ldots, A_{n}(t), \ldots,\right. \\
& \left.\quad A_{1}(t-\tau), \ldots, A_{n}(t-\tau)\right), \quad i=1, \ldots, n, \\
& y_{j}(t)=h_{j}\left(A_{1}(t), \ldots, A_{n}(t)\right), \quad j=1, \ldots, p,
\end{aligned}
$$

where $u_{i}, i=1,2, \ldots, m$ are inputs (or controls); $y_{j}(t), j=$ $1, \ldots, p$ are outputs; $f_{i}, i=1, \ldots, n ; h_{j}, j=1, \ldots, p$ are logical functions.

In this paper, two kinds of inputs (or controls) are considered for (9).

(A) The controls satisfying certain logical rules are called input networks such as

$$
u_{j}(t+1)=g_{j}\left(u_{1}(t), u_{2}(t) \cdots u_{m}(t)\right), \quad j=1, \ldots, m,
$$

where $g_{i}, i=1,2, \ldots, m$ are logical functions, and the initial states $u_{j}(0), j=1,2, \ldots, m$, can be arbitrarily given.

(B) The controls are free Boolean sequences, which means that the controls do not satisfy any logical rule.

Let $u(t)=\ltimes_{j=1}^{m} u_{j}(t), y(t)=\ltimes_{j=1}^{p} y_{j}(t)$. From Lemma 1, for every logical function $f_{i}, g_{j}, h_{l}$, we can find its structure matrix $M_{1 i}, M_{2 j}, M_{3 l}, i=1, \ldots, n, j=1, \ldots, m, l=$ $1, \ldots, p$, respectively. Then from (9) and (10), we can obtain

$$
\begin{gathered}
A_{i}(t+1)=M_{1 i} u(t) x(t) \cdots x(t-\tau), \quad i=1, \ldots, n, \\
u_{j}(t+1)=M_{2 j} u(t), \quad j=1, \ldots, m, \\
y_{l}(t)=M_{3 l} x(t), \quad l=1, \ldots, p .
\end{gathered}
$$

Similar with (7), multiplying (11) yields

$$
\begin{aligned}
x(t+1)= & \ltimes_{i=1}^{n}\left[M_{1 i} u(t) x(t) \cdots x(t-\tau)\right] \\
= & M_{11}\left[\left(I_{2^{m+n(\tau+1)}} \otimes M_{12}\right) \Phi_{m+n(\tau+1)}\right] u(t) x(t) \cdots \\
& \times x(t-\tau) M_{13} \cdots \\
& \times M_{1 n} u(t) x(t) \cdots x(t-\tau) \\
= & \cdots \\
= & M_{11}\left[\ltimes_{i=2}^{n}\left(I_{2^{m+n(\tau+1)}} \otimes M_{1 i} \Phi_{m+n(\tau+1)}\right)\right] u(t) x(t) \cdots \\
& \times x(t-\tau) \\
\triangleq & L u(t) x(t) \cdots x(t-\tau) .
\end{aligned}
$$

And, multiplying (12), it leads to

$$
\begin{aligned}
u(t+1)= & u_{1}(t+1) u_{2}(t+1) \cdots u_{m}(t+1) \\
= & M_{21} u(t) M_{22} u(t) \cdots M_{2 n} u(t) \\
= & M_{21}\left(I_{2^{m}} \otimes M_{22}\right) \Phi_{m}\left(I_{2^{m}} \otimes M_{23}\right) \Phi_{m} \cdots \\
& \times\left(I_{2^{m}} \otimes M_{2 m}\right) \Phi_{m} u(t) \\
\triangleq & G u(t) .
\end{aligned}
$$

Multiplying (13) yields $y(t)=H x(t)$, where $H=$ $M_{31}\left[\ltimes_{l=2}^{p}\left(I_{2^{n}} \otimes M_{3 l} \Phi_{n}\right)\right]$. From the above conclusion, in an algebraic form, a BCN (9) and (10) can be expressed as

$$
\begin{gathered}
x(t+1)=L u(t) x(t) \cdots x(t-\tau), \\
y(t)=H x(t), \\
u(t+1)=G u(t),
\end{gathered}
$$

where $L, H$ are the network transition matrices of two kinds of equations in (9), respectively, and $G$ is the network transition matrix of (10).

Remark 3. It should be noticed that by using the first algebraic form of the network from Chapter 5 of [26], TBCN can be rewritten by a $\mathrm{BCN}$ with no delay. Hence, it can be a good idea to study the observability of TBCNs by using the corresponding BCNs from the results in [10]. However, if the first algebraic form is used, the dimension of network transition matrix of corresponding BCNs will be much bigger which would make computation cost much higher. From (16), it is easy to calculate that the dimension of $L$ is $2^{n} \times 2^{n(\tau+1)+m}$. However, if the TBCNs are rewritten by BCNs using the first algebraic form, then the dimension of the corresponding network transition matrix of the BCNs would be $2^{n(\tau+1)} \times$ $2^{n(\tau+1)+m}$, which is much bigger if $n$ or $\tau$ is a large number. Furthermore, considering the TBCNs directly, we can find the relationship between the network transition matrix (or the Boolean functions) of the TBCN and the state clearly. However, if the $\mathrm{BCN}$ is used, the relationship would not be so clear. 


\section{Observability of Temporal Boolean Control Networks}

In this section, we consider the observability problem of temporal Boolean control network (9), equivalently (16), and the analysis is given via two kinds of controls (A) and (B), respectively.

Definition 4 (see [19]). The temporal Boolean network (16) is observable if for the initial state sequence $x(-i), i \in\{0,1$, $\ldots, \tau\}$, there exists a finite time $s \in \mathbb{N}$, such that the initial state sequence can be uniquely determined by the input controls $u(0), u(1), \ldots, u(s)$ and the outputs $y(0), y(1), \ldots, y(s)$.

For simplicity, we denote the vector $\mathscr{X}(i)=\ltimes_{j=0}^{i} x(-j) \epsilon$ $\Delta_{2^{n(\tau+1)}}, i \in\{0,1, \ldots, \tau\}$.

Definition 5 (see [19]). For temporal Boolean network (16) and control (17) with fixed $G$, the input-state transfer matrix $\mathscr{L}_{i}^{G} \in \mathscr{L}_{2^{n} \times 2^{m+n(\tau+1)}}, i \in \mathbb{N}^{+}$, is defined as follows: for any $u(0) \in \Delta_{2^{m}}$ and any $x(-i) \in \Delta_{2^{n}}, i \in\{0,1, \ldots, \tau\}$, we have

$$
x(i)=\mathscr{L}_{i}^{G} u(0) \mathscr{X}(\tau), \quad i \in \mathbb{N}^{+} .
$$

Now we need a dummy operator to add some fabricated variables when these variables do not appear. Define

$$
\begin{aligned}
E_{n, m} & :=\underbrace{\left[I_{2^{n}} I_{2^{n}} \cdots I_{2^{n}}\right]}_{2^{m n}} \\
& =\delta_{2^{n}} \underbrace{[\underbrace{1,2, \ldots, 2^{n}}, \ldots, \underbrace{1,2, \ldots, 2^{n}}] .}_{2^{m n}} .
\end{aligned}
$$

A straightforward computation shows the following.

Lemma 6. Consider the temporal Boolean network (16),

$$
x(0)=E_{n, \tau} W_{\left[2^{n}, 2^{n \tau}\right]} \mathscr{X}(\tau) .
$$

Proof. Since $\ltimes_{i=1}^{\tau} x(-i) \in \Delta_{2^{n \tau}}$, from the definition of $E_{n, m}$, we have

$$
E_{n, \tau} \ltimes_{i=1}^{\tau} x(-i)=I_{2^{n}}
$$

Hence,

$$
\begin{aligned}
x(0) & =I_{2^{n}} x(0)=E_{n, \tau} \ltimes_{i=1}^{\tau} x(-i) x(0) \\
& =E_{n, \tau} W_{\left[2^{n}, 2^{n \tau}\right]} X(\tau) .
\end{aligned}
$$

4.1. Observability of Input Boolean Networks. We first consider the case that controls satisfy certain logical rules as
(17). Define a sequence of matrices $\mathscr{L}_{s}^{G} \in \mathscr{L}_{2^{n} \times 2^{m+n(\tau+1)}}$ as (23):

$$
\begin{aligned}
& \mathscr{L}_{s}^{G} \\
& :=\left\{\begin{array}{c}
L, \quad s=1, \\
L G\left[\left(I_{2^{m}} \otimes \mathscr{L}_{1}^{G}\right) \Phi_{m}\right]\left[I_{2^{m}} \otimes W_{2^{n \tau}, 2^{n(\tau+1)}} \Phi_{n(\tau)}\right], \\
s=2, \\
L G^{s-1}\left[\left(I_{2^{m}} \otimes \mathscr{L}_{s-1}^{G}\right) \Phi_{m}\right]\left[\ltimes_{i=s-2}^{1} \mathscr{M}_{i}^{G}\right] \\
\ltimes\left[I_{2^{m}} \otimes W_{\left[2^{n(\tau-s+2)}, 2^{n(\tau+1)}\right]} \Phi_{n(\tau-s+2)}\right], \\
s=3, \ldots, \tau+1, \\
L G^{s-1}\left[\left(I_{2^{m}} \otimes \mathscr{L}_{s-1}^{G}\right) \Phi_{m}\right]\left[\ltimes_{i=s-2}^{s-\tau-1} \mathscr{M}_{i}^{G}\right], \\
s>\tau+1,
\end{array}\right.
\end{aligned}
$$

where $\mathscr{M}_{i}^{G}=I_{2^{m+n(\tau+1)}} \otimes \mathscr{L}_{i}^{G} \Phi_{m+n(\tau+1)}$ and $\mathscr{H}_{0}^{G}=$ $H E_{n, \tau} W_{\left[2^{n}, 2^{n \tau}\right]}, \mathscr{H}_{s}^{G}=H \mathscr{L}_{s}^{G}, s \in \mathbb{N}^{+}$, and the transition matrices $L, G$, and $H$ are defined in (16) and (17). Furthermore, we split $\mathscr{H}_{j}^{G} \in \mathscr{L}_{2^{p} \times 2^{m+n(\tau+1)}}, j \in \mathbb{N}^{+}$, into $2^{m}$ equal blocks as $\mathscr{H}_{j}^{G}=\left[\mathscr{H}_{j, 1}^{G}, \mathscr{H}_{j, 2}^{G}, \ldots, \mathscr{H}_{j, 2^{m}}^{G}\right]$ with each $\mathscr{H}_{j, i}^{G} \in \mathscr{L}_{2^{p} \times 2^{n(\tau+1)}}$, $i=1,2, \ldots, 2^{m}, j \in \mathbb{N}^{+}$.

Theorem 7. Consider the temporal Boolean network (16) with control (17). Assume that $u(0)=\delta_{2^{m}}^{i}, i \in\left\{1,2, \ldots, 2^{m}\right\}$. Then, (16) and (17) are observable if and only if there exists a finite time s such that $\operatorname{rank}\left(\mathcal{O}_{1, i, s}\right)=2^{n(\tau+1)}$, where

$$
\mathcal{O}_{1, i, s}=\left[\begin{array}{c}
\mathscr{H}_{0}^{G} \\
\mathscr{H}_{1, i}^{G} \\
\vdots \\
\mathscr{H}_{s, i}^{G}
\end{array}\right] .
$$

Proof. Firstly, from Lemma 6 and (16),

$$
y(0)=H x(0)=H E_{n, \tau} W_{\left[2^{n}, 2^{n \tau}\right]} \mathscr{X}(\tau) \triangleq \mathscr{H}_{0}^{G} \mathscr{X}(\tau) .
$$

Since $u(0)=\delta_{2^{m}}^{i}$, we have from (18) that

$y(1)$

$$
\begin{aligned}
& =H x(1)=H L u(0) \mathscr{X}(\tau) \\
& \triangleq H \mathscr{L}_{1}^{G} u(0) \mathscr{X}(\tau)=\mathscr{H}_{1, i}^{G} \mathscr{X}(\tau),
\end{aligned}
$$

$y(2)$

$$
\begin{aligned}
& =H L u(1) x(1) \mathscr{X}(\tau-1) \\
& =H L G u(0) \mathscr{L}_{1}^{G} u(0) \mathscr{X}(\tau) \mathscr{X}(\tau-1) \\
& =H L G\left[\left(I_{2^{m}} \otimes \mathscr{L}_{1}^{G}\right) \Phi_{m}\right] u(0) \mathscr{X}(\tau) \mathscr{X}(\tau-1) \\
& =H L G\left[\left(I_{2^{m}} \otimes \mathscr{L}_{1}^{G}\right) \Phi_{m}\right] u(0) W_{\left[2^{n \tau}, 2^{n(\tau+1)}\right]} \Phi_{n \tau} \mathscr{X}(\tau)
\end{aligned}
$$




$$
\begin{aligned}
= & H L G\left[\left(I_{2^{m}} \otimes \mathscr{L}_{1}^{G}\right) \Phi_{m}\right] \\
& \times\left[I_{2^{m}} \otimes W_{\left[2^{n \tau}, 2^{n(\tau+1)}\right]} \Phi_{n \tau}\right] u(0) \mathscr{X}(\tau) \\
\triangleq & H \mathscr{L}_{2}^{G} u(0) \mathscr{X}(\tau)=\mathscr{H}_{2, i}^{G} \mathscr{X}(\tau),
\end{aligned}
$$

$y(3)$

$$
\begin{aligned}
& =H L u(2) x(2) x(1) \mathscr{X}(\tau-2) \\
& =H L G^{2} u(0) \mathscr{L}_{2}^{G} u(0) \mathscr{X}(\tau) \mathscr{L}_{1}^{G} u(0) \mathscr{X}(\tau) \mathscr{X}(\tau-2) \\
& =H L G^{2}\left[\left(I_{2^{m}} \otimes \mathscr{L}_{2}^{G}\right) \Phi_{m}\right] \\
& \times u(0) \mathscr{X}(\tau) \mathscr{L}_{1}^{G} u(0) \mathscr{X}(\tau) \mathscr{X}(\tau-2) \\
& =H L G^{2}\left[\left(I_{2^{m}} \otimes \mathscr{L}_{2}^{G}\right) \Phi_{m}\right] \\
& \times\left[\left(I_{2^{m+n(\tau+1)}} \otimes \mathscr{L}_{1}^{G}\right) \Phi_{m+n(\tau+1)}\right] \\
& \times u(0) \mathscr{X}(\tau) \mathscr{X}(\tau-2) \\
& =H L G^{2}\left[\left(I_{2^{m}} \otimes \mathscr{L}_{2}^{G}\right) \Phi_{m}\right] \\
& \times\left[\left(I_{2^{m+n(\tau+1)}} \otimes \mathscr{L}_{1}^{G}\right) \Phi_{m+n(\tau+1)}\right] \\
& \times\left[I_{2^{m}} \otimes W_{\left[2^{n(\tau-1)}, 2^{n(\tau+1)}\right]} \Phi_{n(\tau-1)}\right] u(0) \mathscr{X}(\tau) \\
& \triangleq H \mathscr{L}_{3}^{G} u(0) \mathscr{X}(\tau)=\mathscr{H}_{3, i}^{G} \mathscr{X}(\tau) \text {, } \\
& \vdots \\
& y(\tau+1) \\
& =H L u(\tau) x(\tau) \cdots x(1) \mathscr{X}(0) \\
& =H L G^{\tau} u(0)\left[\ltimes_{i=\tau}^{1} \mathscr{L}_{i}^{G} u(0) \mathscr{X}(\tau)\right] \mathscr{X}(0) \\
& =H L G^{\tau}\left[\left(I_{2^{m}} \otimes \mathscr{L}_{\tau}^{G}\right) \Phi_{m}\right]\left[\ltimes_{i=\tau-1}^{1} \mathscr{M}_{i}^{G}\right] \\
& \times\left[I_{2^{m}} \otimes W_{\left[2^{n}, 2^{n(\tau+1)}\right]} \Phi_{n}\right] u(0) \mathscr{X}(\tau) \\
& \triangleq H \mathscr{L}_{\tau+1}^{G} u(0) \mathscr{X}(\tau)=\mathscr{H}_{\tau+1, i}^{G} \mathscr{X}(\tau) .
\end{aligned}
$$

For $s>\tau+1$, we can obtain that

$$
\begin{aligned}
y(\tau+2) & \\
= & H L u(\tau+1) x(\tau+1) \cdots x(1) \\
= & H L G^{\tau+1} u(0)\left[\ltimes_{i=\tau+1}^{1} \mathscr{L}_{i}^{G} u(0) \mathscr{X}(\tau)\right] \\
= & H L G^{\tau+1}\left[\left(I_{2^{m}} \otimes \mathscr{L}_{\tau+1}^{G}\right) \Phi_{m}\right] \\
& \times\left[\ltimes_{i=\tau}^{1} \mathscr{M}_{i}^{G}\right] u(0) \mathscr{X}(\tau) \\
\triangleq & H \mathscr{L}_{\tau+2}^{G} u(0) \mathscr{X}(\tau)=\mathscr{H}_{\tau+2, i}^{G} \mathscr{X}(\tau),
\end{aligned}
$$

$$
\begin{aligned}
y(\tau+3) & \\
= & H L u(\tau+2) x(\tau+2) \cdots x(2) \\
= & H L G^{\tau+2} u(0)\left[\ltimes_{i=\tau+2}^{2} \mathscr{L}_{i}^{G} u(0) \mathscr{X}(\tau)\right] \\
= & H L G^{\tau+2}\left[\left(I_{2^{m}} \otimes \mathscr{L}_{\tau+2}^{G}\right) \Phi_{m}\right] \\
& \times\left[\ltimes_{i=\tau+1}^{2} \mathscr{M}_{i}^{G}\right] u(0) \mathscr{X}(\tau) \\
\triangleq & H \mathscr{L}_{\tau+3}^{G} u(0) \mathscr{X}(\tau)=\mathscr{H}_{\tau+3, i}^{G} \mathscr{X}(\tau), \\
& \vdots \\
y(s) & \\
= & H L u(s-1) x(s-1) \cdots x(s-\tau-1) \\
= & H L G^{s-1} u(0)\left[\ltimes_{i=s-2}^{s-\tau-1} \mathscr{L}_{i}^{G} u(0) \mathscr{X}(\tau)\right] \\
= & H L G^{s-1}\left[\left(I_{2^{m}} \otimes \mathscr{L}_{s-1}^{G}\right) \Phi_{m}\right] \\
& \times\left[\ltimes_{i=s-2}^{s-\tau-1} \mathscr{M}_{i}^{G}\right] u(0) \mathscr{X}(\tau) \\
\triangleq & H \mathscr{L}_{s}^{G} u(0) \mathscr{X}(\tau)=\mathscr{H}_{s, i}^{G} \mathscr{X}(\tau) .
\end{aligned}
$$

From the above analysis, and definition of $\mathcal{O}_{1, i, s}$ in (24), we can see that

$$
\mathcal{O}_{1, i, s} \mathscr{X}(\tau)=\left[\begin{array}{c}
y(0) \\
y(1) \\
\vdots \\
y(s)
\end{array}\right] .
$$

Since $\mathscr{X}(\tau) \in \Delta_{2^{n(\tau+1)}}, \mathcal{O}_{1, i, s} \mathscr{X}(\tau) \in \operatorname{Col}\left(\mathcal{O}_{1, i, s}\right)$. It implies that $\mathscr{X}(\tau)$ is determined uniquely by the outputs $y(0), \ldots, y(s)$ if and only if there exist no similar elements in $\operatorname{Col}\left(\mathcal{O}_{1, i, s}\right)$, or equivalently, there are no equal columns in $\mathcal{O}_{1, i, s}$, that is, $\operatorname{rank}\left(\mathcal{O}_{1, i, s}\right)=2^{n(\tau+1)}$. The proof is completed.

Corollary 8. Consider the temporal Boolean network (16) with control (17). Equations (16) and (17) are observable if and only if there exist a finite time $s$ and $i \in\left\{1,2, \ldots, 2^{m}\right\}$ such that $\operatorname{rank}\left(\mathcal{O}_{1, i, s}\right)=2^{n(\tau+1)}$.

Remark 9. When the time delay $\tau=0$, then the temporal Boolean control network (16) and (17) become a Boolean control network. In this case, it can be induced from (23) that

$$
\mathscr{L}_{s}^{G}= \begin{cases}L, & s=1, \\ L G^{s-1}\left[\left(I_{2^{m}} \otimes \mathscr{L}_{s-1}^{G}\right) \Phi_{m}\right], & s>1 .\end{cases}
$$

Then, the observability of the BCN with input Boolean network controls can be deduced from Theorem 7 and Corollary 8.

4.2. Control via Free Boolean Sequence. In the following, the case where the controls are free Boolean sequences is 
considered. We split $L$ given in (16) into $2^{m}$ equal blocks as

$$
L=\left[L_{1}, L_{2}, \ldots, L_{2^{m}}\right]
$$

with each $L_{i} \in \mathscr{L}_{2^{n} \times 2^{n(\tau+1)}}, i=1,2, \ldots, 2^{m}$. Define a sequence of matrices $\widetilde{\mathscr{L}}_{s, i_{s-1}, \ldots, i_{0}} \in \mathscr{L}_{2^{n} \times 2^{n(\tau+1)}}, s \in \mathbb{N}^{+}, i_{s-1} \in\{1,2$, $\ldots, 2^{m}$ as (31):

$$
\begin{aligned}
& \widetilde{\mathscr{L}}_{s, i_{s-1}, \ldots, i_{0}} \\
& = \begin{cases}L_{i_{0}}, & s=1, \\
L_{i_{1}} L_{i_{0}} W_{\left[2^{n \tau}, 2^{n(\tau+1)}\right]} \Phi_{n \tau}, & s=2, \\
L_{i_{s-1}} \widetilde{\mathscr{L}}_{s-1, i_{s-2}, \ldots, i_{0}}\left[\ltimes_{j=s-2}^{1} \widetilde{M}_{j}\right] & \\
\ltimes W_{\left[2^{n(\tau-s+2)}, 2^{n(\tau+1)}\right]} \Phi_{n(\tau-s+2)}, & s=3, \ldots, \tau+1, \\
L_{i_{s-1}} \widetilde{\mathscr{L}}_{s-1, i_{s-2}, \ldots, i_{0}}\left[\ltimes_{j=s-2}^{s-\tau-1} \widetilde{\mathscr{M}}_{j}\right], & s>\tau+1,\end{cases}
\end{aligned}
$$

where $\widetilde{\mathscr{M}}_{j}=I_{2^{n(\tau+1)}} \otimes \widetilde{\mathscr{L}}_{j, i_{j-1}, \ldots, i_{0}} \Phi_{n(\tau+1)}$, the transition matrices $L, G$, and $H$ are defined in (16) and (17).

Theorem 10. Consider the temporal Boolean network (16). Assume that the controls are free Boolean sequences with $u(l)=$ $\delta_{2^{m}}^{i_{l}}, l \in \mathbb{N}, i_{l} \in\left\{1,2, \ldots, 2^{m}\right\}$. Then, (16) is observable if and only if there exists a finite time s such that $\operatorname{rank}\left(\mathcal{O}_{2, s}\right)=2^{n(\tau+1)}$, where

$$
\mathcal{O}_{2, s}=\left[\begin{array}{c}
\mathscr{H}_{0}^{G} \\
H \widetilde{\mathscr{L}}_{1, i_{0}} \\
H \widetilde{\mathscr{L}}_{2, i_{1}, i_{0}} \\
\vdots \\
H \widetilde{\mathscr{L}}_{s, i_{s-1}, \ldots, i_{0}}
\end{array}\right] .
$$

Proof. Since the controls are free Boolean sequences with $u(l)=\delta_{2^{m}}^{i_{l}}, l \in \mathbb{N}, i_{l} \in\left\{1,2, \ldots, 2^{m}\right\}$, from (16) we have

$y(1)$

$$
\begin{aligned}
& =H x(1)=H L u(0) \mathscr{X}(\tau) \\
& =H L_{i_{0}} \mathscr{X}(\tau) \triangleq H \widetilde{\mathscr{L}}_{1, i_{0}} \mathscr{X}(\tau),
\end{aligned}
$$

$y(2)$

$$
\begin{aligned}
& =H x(2)=H L u(1) x(1) \mathscr{X}(\tau-1) \\
& =H L u(1) \operatorname{Lu}(0) \mathscr{X}(\tau) \mathscr{X}(\tau-1) \\
& =H L u(1) \operatorname{Lu}(0) W_{\left[2^{n \tau}, 2^{n(\tau+1)}\right]} \Phi_{n \tau} \mathscr{X}(\tau)
\end{aligned}
$$

$$
\begin{aligned}
& =H L_{i_{1}} L_{i_{0}} W_{\left[2^{n \tau}, 2^{n(\tau+1)}\right]} \Phi_{n \tau} \mathscr{X}(\tau) \\
& \triangleq H \widetilde{\mathscr{L}}_{2, i_{1}, i_{0}} \mathscr{X}(\tau)
\end{aligned}
$$

$y(3)$

$$
\begin{aligned}
= & H x(3)=H L u(2) x(2) x(1) \mathscr{X}(\tau-2) \\
= & H L u(2) \widetilde{\mathscr{L}}_{2, i_{1}, i_{0}} \mathscr{X}(\tau) \widetilde{\mathscr{L}}_{1, i_{0}} \mathscr{X}(\tau) \mathscr{X}(\tau-2) \\
= & H L_{i_{2}} \widetilde{\mathscr{L}}_{2, i_{1}, i_{0}}\left[\left(I_{2^{n(\tau+1)}} \otimes \widetilde{\mathscr{L}}_{1, i_{0}}\right) \Phi_{n(\tau+1)}\right] \\
& \times \mathscr{X}(\tau) \mathscr{X}(\tau-2) \\
= & H L_{i_{2}} \widetilde{\mathscr{L}}_{2, i_{1}, i_{0}}\left[\left(I_{2^{n(\tau+1)}} \otimes \widetilde{\mathscr{L}}_{1, i_{0}}\right) \Phi_{n(\tau+1)}\right] \\
& \times\left[W_{\left[2^{n(\tau-1)}, 2^{n(\tau+1)}\right]} \Phi_{n(\tau-1)}\right] \mathscr{X}(\tau) \\
\triangleq & H \widetilde{\mathscr{L}}_{3, i_{2}, i_{1}, i_{0}} \mathscr{X}(\tau), \\
& \vdots \\
y(\tau+ & 1) \\
= & H L u(\tau) x(\tau) \cdots x(1) \mathscr{X}_{(0)} \\
= & H L u(\tau)\left[\ltimes_{j=\tau}^{1} \widetilde{\mathscr{L}}_{j, i_{j-1}, \ldots, i_{0}} \mathscr{X}(\tau)\right] \mathscr{X}(0) \\
= & H L_{i_{\tau}} \widetilde{\mathscr{L}}_{\tau, i_{\tau-1}, \ldots, i_{0}}\left[\ltimes_{j=\tau-1}^{1} \widetilde{\mathscr{M}}_{j}\right] \\
& \times W_{\left[2^{n}, 2^{n(\tau+1)}\right]} \Phi_{n} \mathscr{X}(\tau) \\
\triangleq & H \widetilde{\mathscr{L}}_{\tau+1, i_{\tau}, \ldots, i_{0}} \mathscr{X}(\tau) . \\
& \\
& \\
& \\
&
\end{aligned}
$$

For $s>\tau+1$, we can obtain that

$$
\begin{aligned}
y(\tau+ & 2) \\
& =H \operatorname{Lu}(\tau+1) x(\tau+1) \cdots x(1) \\
& =H L u(\tau+1)\left[\ltimes_{j=\tau+1}^{1} \widetilde{\mathscr{L}}_{j, i_{j-1}, \ldots, i_{0}} \mathscr{X}(\tau)\right] \\
& =H L_{i_{\tau+1}} \widetilde{\mathscr{L}}_{\tau+1, i_{\tau}, \ldots, i_{0}}\left[\ltimes_{i=\tau}^{1} \widetilde{\mathscr{M}}_{j}\right] \mathscr{X}(\tau) \\
& \triangleq H \widetilde{\mathscr{L}}_{\tau+2, i_{\tau+1}, \ldots, i_{0}} \mathscr{X}(\tau), \\
y(\tau+ & 3) \\
& =H L u(\tau+2) x(\tau+2) \cdots x(2) \\
& =H L u(\tau+2)\left[\ltimes_{j=\tau+2}^{2} \widetilde{\mathscr{L}}_{j, i_{j-1}, \ldots, i_{0}} \mathscr{X}(\tau)\right] \\
& =H L_{i_{\tau+2}} \widetilde{\mathscr{L}}_{\tau+2, i_{\tau+1}, \ldots, i_{0}}\left[\ltimes_{i=\tau+1}^{2} \widetilde{\mathscr{M}}_{j}\right] \mathscr{X}(\tau) \\
& \triangleq H \widetilde{\mathscr{L}}_{\tau+3, i_{\tau+2}, \ldots, i_{0}} \mathscr{X}(\tau),
\end{aligned}
$$


$y(s)$

$$
\begin{aligned}
& =H \operatorname{Lu}(s-1) x(s-1) \cdots x(s-\tau-1) \\
& =H \operatorname{Lu}(s-1)\left[\ltimes_{i=s-2}^{s-\tau-1} \widetilde{\mathscr{L}}_{j, i_{j-1}, \ldots, i_{0}} \mathscr{X}(\tau)\right] \\
& =H L_{i_{s-1}} \widetilde{\mathscr{L}}_{s-1, i_{s-2}, \ldots, i_{0}}\left[\ltimes_{i=s-2}^{s-\tau-1} \widetilde{\mathscr{M}}_{j}\right] \mathscr{X}(\tau) \\
& \triangleq H \widetilde{\mathscr{L}}_{s, i_{s-1}, \ldots, i_{0}} \mathscr{X}(\tau) .
\end{aligned}
$$

Thus, from (25) and the definition of $\mathcal{O}_{2, s}$ in (32), we can see that

$$
\mathcal{O}_{2, s} \mathscr{X}(\tau)=\left[\begin{array}{c}
y(0) \\
y(1) \\
\vdots \\
y(s)
\end{array}\right] .
$$

Similar with the proof of Theorem 7 , we conclude that $\mathscr{X}(\tau)$ can be determined uniquely by the outputs $y(0), \ldots, y(s)$ if and only if $\operatorname{rank}\left(\mathcal{O}_{2, s}\right)=2^{n(\tau+1)}$. The proof is completed.

Corollary 11. Consider the temporal Boolean network (16). The system (16) is observable if and only if there exists a finite time $s$ and a sequence $i_{0}, i_{1}, \ldots, i_{s-1} \in\left\{1,2, \ldots, 2^{m}\right\}$ such that $\operatorname{rank}\left(\mathcal{O}_{2, s}\right)=2^{n(\tau+1)}$.

Remark 12. As a special case, when $\tau=0$, then from the proof of Theorem 10, we have $\mathscr{H}_{0}^{G}=H$, and

$$
\begin{gathered}
\widetilde{\mathscr{L}}_{1, i_{0}}=L_{i_{0}}, \\
\widetilde{\mathscr{L}}_{s+1, i_{s}, \ldots, i_{0}}=L_{i_{s+1}} \widetilde{\mathscr{L}}_{s, i_{s-1}, \ldots, i_{0}}, \quad s>0 .
\end{gathered}
$$

Then, Corollary 11 is equivalent with Theorem 26 in [8] for the observability of BCNs.

Remark 13. For Theorems 7 and 10, when $\tau=1$, the third explicit expressions of $\mathscr{L}_{s}^{G}$ in $(23)$ and $\widetilde{\mathscr{L}}_{s, i_{s-1}, \ldots, i_{0}}$ in (31) for $s=3, \ldots, \tau+1$ should be omitted.

\section{An Example}

Given logical arguments $P, Q \in \Delta$, we have the following structure matrices for the fundamental logical functions: $\neg P=M_{n} P, P \vee Q=M_{d} P Q, P \wedge Q=M_{c} P Q, P \rightarrow Q=M_{i} P Q$, $P \leftrightarrow Q=M_{e} P Q$, where $M_{n}=\delta_{2}[2,1], M_{d}=\delta_{2}[1,1,1,2]$, $M_{c}=\delta_{2}[1,2,2,2], M_{i}=\delta_{2}[1,2,1,1], M_{e}=\delta_{2}[1,2,2,1]$.

Example 14. Consider the following temporal Boolean network:

$$
\begin{gathered}
A(t+1)=u(t) \vee A(t) \longrightarrow A(t-1) \longleftrightarrow A(t-2), \\
y(t)=\neg A(t) .
\end{gathered}
$$

Let $x(t)=A(t)$, it is easy to get $H=M_{n}, L=M_{e} M_{i} M_{d}$, and $\tau=2$.

(A) When the controls satisfy the logical rule

$$
u(t+1)=\neg u(t),
$$

then the transition matrix $G=M_{n}$. Now, assume that $u(0)=$ $\delta_{2}^{1}$, by calculation, we have

$$
\begin{aligned}
& \mathscr{H}_{0}^{G}=\delta_{2}[2,2,2,2,1,1,1,1], \\
& \mathscr{H}_{1,1}^{G}=\delta_{2}[2,1,1,2,2,1,1,2], \\
& \mathscr{H}_{2,1}^{G}=\delta_{2}[2,2,2,2,1,2,2,1] \text {, } \\
& \mathscr{H}_{3,1}^{G}=\delta_{2}[2,1,1,2,2,1,1,2] \text {, } \\
& \mathscr{H}_{4,1}^{G}=\delta_{2}[2,1,1,2,1,1,1,1], \\
& \mathscr{H}_{5,1}^{G}=\delta_{2}[2,1,1,2,1,1,1,1] \text {, } \\
& \mathcal{O}_{1,1, s}=\left[\begin{array}{c}
\mathscr{H}_{0}^{G} \\
\mathscr{H}_{1,1}^{G} \\
\mathscr{H}_{2,1}^{G} \\
\mathscr{H}_{3,1}^{G} \\
\mathscr{H}_{4,1}^{G} \\
\mathscr{H}_{5,1}^{G} \\
\vdots
\end{array}\right] \\
& =\left[\begin{array}{llllllll}
2 & 2 & 2 & 2 & 1 & 1 & 1 & 1 \\
2 & 1 & 1 & 2 & 2 & 1 & 1 & 2 \\
2 & 2 & 2 & 2 & 1 & 2 & 2 & 1 \\
2 & 1 & 1 & 2 & 2 & 1 & 1 & 2 \\
2 & 1 & 1 & 2 & 1 & 1 & 1 & 1 \\
2 & 1 & 1 & 2 & 1 & 1 & 1 & 1 \\
\vdots & & & & & & &
\end{array}\right] .
\end{aligned}
$$

Hence, for any $s>0$, there are only 4 linearly independent columns, which means that $\operatorname{rank}\left(\mathcal{O}_{1,1, s}\right)<2^{n(\tau+1)}=8$ for any $s>0$, and the system is not observable from Theorem 7 . Similarly, if $u(0)=\delta_{2}^{2}$, we have the same conclusion.

(B) When controls are free sequences with $u(0)=\delta_{2}^{1}$, $u(i)=\delta_{2}^{2}, i=1,2, \ldots$. By calculation, it leads to

$$
\begin{aligned}
\mathscr{H}_{0}^{G} & =\delta_{2}[2,2,2,2,1,1,1,1], \\
H \widetilde{\mathscr{L}}_{1,1} & =\delta_{2}[2,1,1,2,2,1,1,2], \\
H \widetilde{\mathscr{L}}_{2,2,1} & =\delta_{2}[2,2,1,1,1,2,1,2], \\
H \widetilde{\mathscr{L}}_{3,2,2,1} & =\delta_{2}[2,1,2,2,1,2,1,1], \\
H \widetilde{\mathscr{L}}_{4,2,2,2,1} & =\delta_{2}[2,1,2,1,2,1,1,2],
\end{aligned}
$$


and hence,

$$
\sigma_{2, s}=\left[\begin{array}{c}
\mathscr{H}_{0}^{G} \\
H \widetilde{\mathscr{L}}_{1,1} \\
H \widetilde{\mathscr{L}}_{2,2,1} \\
H \widetilde{\mathscr{L}}_{3,2,2,1} \\
H \widetilde{\mathscr{L}}_{4,2,2,2,1} \\
\vdots
\end{array}\right]=\left[\begin{array}{llllllll}
2 & 2 & 2 & 2 & 1 & 1 & 1 & 1 \\
2 & 1 & 1 & 2 & 2 & 1 & 1 & 2 \\
2 & 2 & 1 & 1 & 1 & 2 & 1 & 2 \\
2 & 1 & 2 & 2 & 1 & 2 & 1 & 1 \\
2 & 1 & 2 & 1 & 2 & 1 & 1 & 2 \\
\vdots & & & & & & &
\end{array}\right] .
$$

When $s=2$, it is enough to see that there are no equal columns in $\mathcal{O}_{2,2}$. So, the system is observable by Theorem 10 .

From cases (A) and (B), it is easy to notice that the selection of controls is very important for the observability of the temporal Boolean control network.

\section{Conclusion}

In this brief paper, necessary and sufficient conditions for the observability of temporal Boolean control networks have been derived. By using semi-tensor product of matrices and the matrix expression of logic, we have converted the temporal Boolean control networks into discrete systems with time delays. Moreover, the observability has been investigated via two different kinds of controls. Finally, an example has been given to show the efficiency of the proposed results.

\section{Acknowledgments}

The authors would like to take this opportunity to thank the reviewers for their constructive comments and useful suggestions. This work was partially supported by the NNSF of China (Grants nos. 11101373, 11271333, and 61074011).

\section{References}

[1] S. A. Kauffman, "Metabolic stability and epigenesis in randomly constructed genetic nets," Journal of Theoretical Biology, vol. 22, no. 3, pp. 437-467, 1969.

[2] S. Kauffman, The Origins of Order: Self-Organization and Selection in Evolution, Oxford University Press, New York, NY, USA, 1993.

[3] S. Kauffman, At Home in the Universe, Oxford University Press, New York, NY, USA, 1995.

[4] D. Cheng, "Semi-tensor product of matrices and its applications: a survey," in Proceedings of the 4th International Congress of Chinese Mathematicians, pp. 641-668, Hangzhou, China, December 2007.

[5] S. Huang and D. E. Ingber, "Shape-dependent control of cell growth, differentiation, and apoptosis: Switching between attractors in cell regulatory networks," Experimental Cell Research, vol. 261, no. 1, pp. 91-103, 2000.

[6] D. Cheng, "Input-state approach to Boolean networks," IEEE Transactions on Neural Networks, vol. 20, no. 3, pp. 512-521, 2009.
[7] D. Cheng, Z. Li, and H. Qi, "Realization of Boolean control networks," Automatica, vol. 46, no. 1, pp. 62-69, 2010.

[8] D. Cheng and H. Qi, "Controllability and observability of Boolean control networks," Automatica, vol. 45, no. 7, pp. 16591667, 2009.

[9] D. Cheng and H. Qi, "A linear representation of dynamics of Boolean networks," IEEE Transactions on Automatic Control, vol. 55, no. 10, pp. 2251-2258, 2010.

[10] D. Laschov and M. Margaliot, "Controllability of Boolean control networks via the Perron-Frobenius theory," Automatica, vol. 48, no. 6, pp. 1218-1223, 2012.

[11] D. Laschov and M. Margaliot, "A maximum principle for singleinput Boolean control networks," IEEE Transactions on Automatic Control, vol. 56, no. 4, pp. 913-917, 2011.

[12] D. Laschov and M. Margaliot, "A pontryagin maximum principle for multi-input boolean control networks," in Recent Advances in Dynamics and Control of Neural Networks, Cambridge Scientific Publishers, 2011.

[13] Z.-H. Guan, T.-H. Qian, and X. Yu, "Controllability and observability of linear time-varying impulsive systems," IEEE Transactions on Circuits and Systems I, vol. 49, no. 8, pp. 1198-1208, 2002.

[14] Y. Liu and S. Zhao, "Controllability for a class of linear timevarying impulsive systems with time delay in control input," IEEE Transactions on Automatic Control, vol. 56, no. 2, pp. 395399, 2011.

[15] Y. Liu and S. Zhao, "Controllability analysis of linear time-varying systems with multiple time delays and impulsive effects," Nonlinear Analysis: Real World Applications, vol. 13, no. 2, pp. 558-568, 2012.

[16] G. Xie and L. Wang, "Necessary and sufficient conditions for controllability and observability of switched impulsive control systems," IEEE Transactions on Automatic Control, vol. 49, no. 6, pp. 960-966, 2004.

[17] S. Zhao and J. Sun, "Controllability and observability for timevarying switched impulsive controlled systems," International Journal of Robust and Nonlinear Control, vol. 20, no. 12, pp. 1313$1325,2010$.

[18] S. Zhao and J. Sun, "A geometric approach for reachability and observability of linear switched impulsive systems," Nonlinear Analysis, vol. 72, no. 11, pp. 4221-4229, 2010.

[19] F. Li, J. Sun, and Q. Wu, "Observability of boolean control networks with state time delays," IEEE Transactions on Neural Networks, vol. 22, no. 6, pp. 948-954, 2011.

[20] J. Lu, D. W. C. Ho, and J. Kurths, "Consensus over directed static networks with arbitrary finite communication delays," Physical Review E, vol. 80, no. 6, Article ID 066121, 2009.

[21] J. Lu, D. W. C. Ho, and J. Cao, "Synchronization in an array of nonlinearly coupled chaotic neural networks with delay coupling," International Journal of Bifurcation and Chaos in Applied Sciences and Engineering, vol. 18, no. 10, pp. 3101-3111, 2008.

[22] S. Lyu, "Combining boolean method with delay times for determining behaviors of biological networks," in Proceedings of the 31st Annual International Conference of the IEEE Engineering in Medicine and Biology Society, pp. 4884-4887, Minneapolis, Minn, USA, September 2009.

[23] C. Cotta, "On the evolutionary inference of temporal boolean networks," in Computational Methods in Neural Modeling, vol. 2686 of Lecture Notes in Computer Science, pp. 494-501, 2003.

[24] C. Fogelberg and V. Palade, "Machine learning and genetic regulatory networks: a review and a roadmap," in Foundations of Computational, Intelligence, vol. 201 of Studies in Computational Intelligence, pp. 3-34, 2009. 
[25] A. Silvescu and V. Honavar, "Temporal Boolean network models of genetic networks and their inference from gene expression time series," Complex Systems, vol. 13, no. 1, pp. 61-78, 2001.

[26] D. Cheng, H. Qi, and Z. Li, Analysis and Control of Boolean Networks: A Semi-Tensor Product Approach, Springer, New York, NY, USA, 2011.

[27] Y. Liu, J. Lu, and B. Wu, "Some necessary and sufficient conditions for the outputcontrollability of temporal Boolean control networks," ESAIM: Control, Optimization and Calculus of Variations. In press.

[28] Y. Liu, H. Chen, and B. Wu, "Controllability of Boolean control networks with impulsive effects and forbidden states," Mathematical Methods in the Applied Sciences, 2013. 


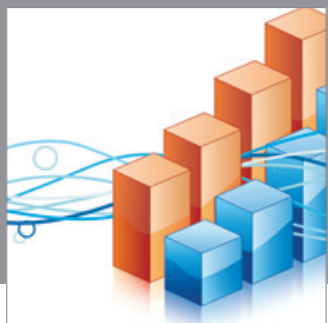

Advances in

Operations Research

mansans

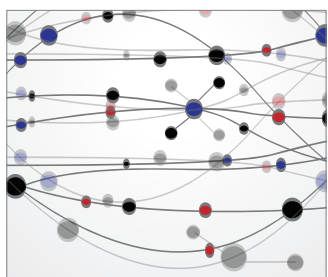

The Scientific World Journal
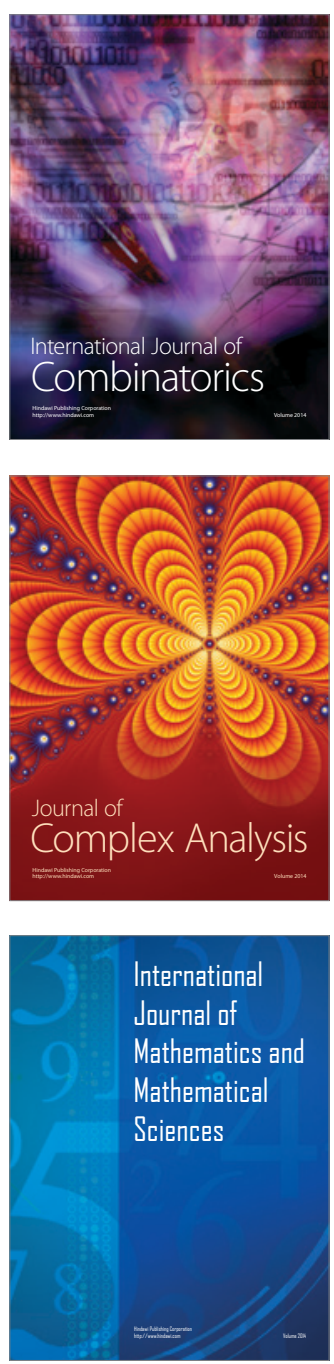
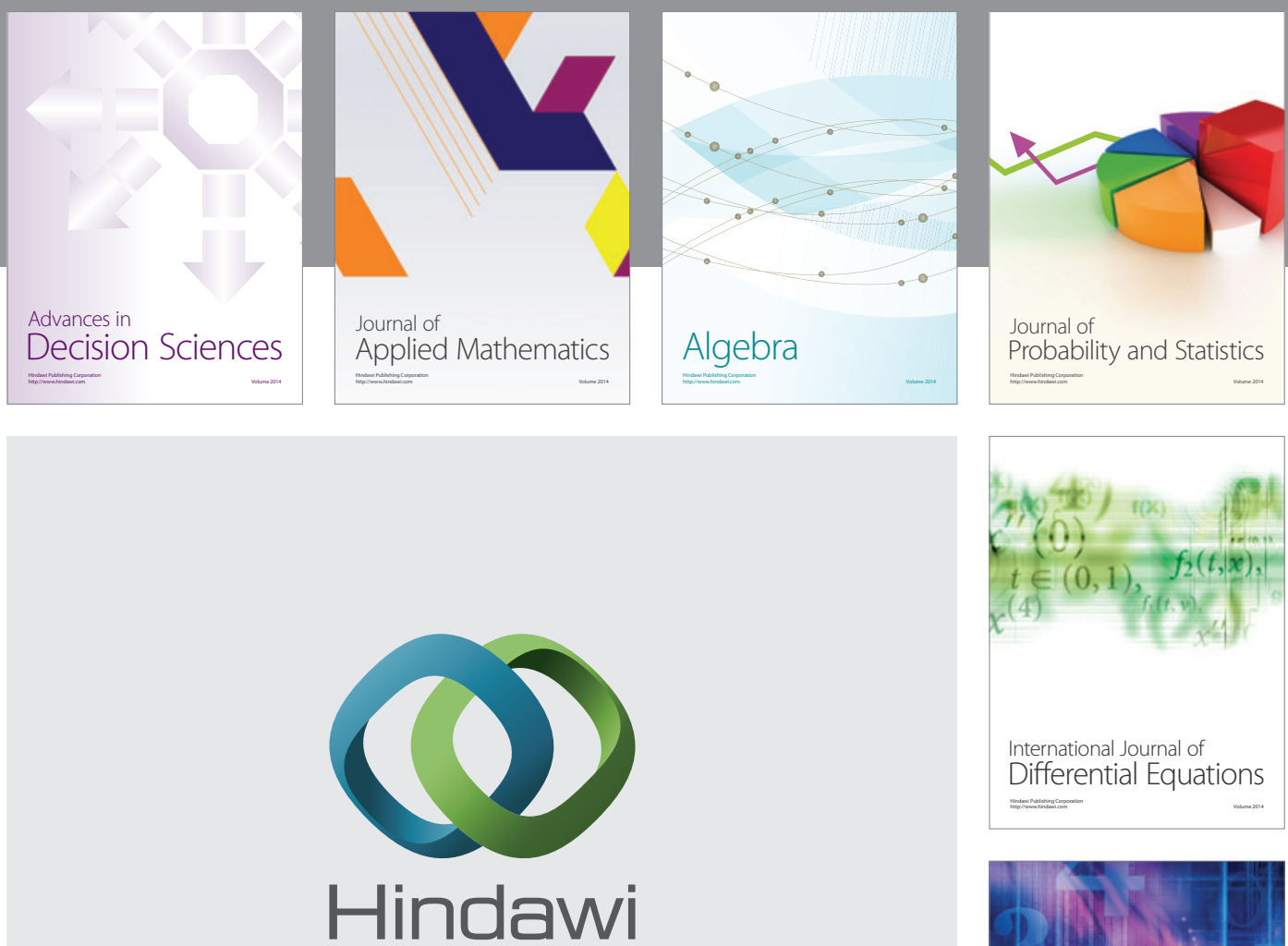

Submit your manuscripts at http://www.hindawi.com
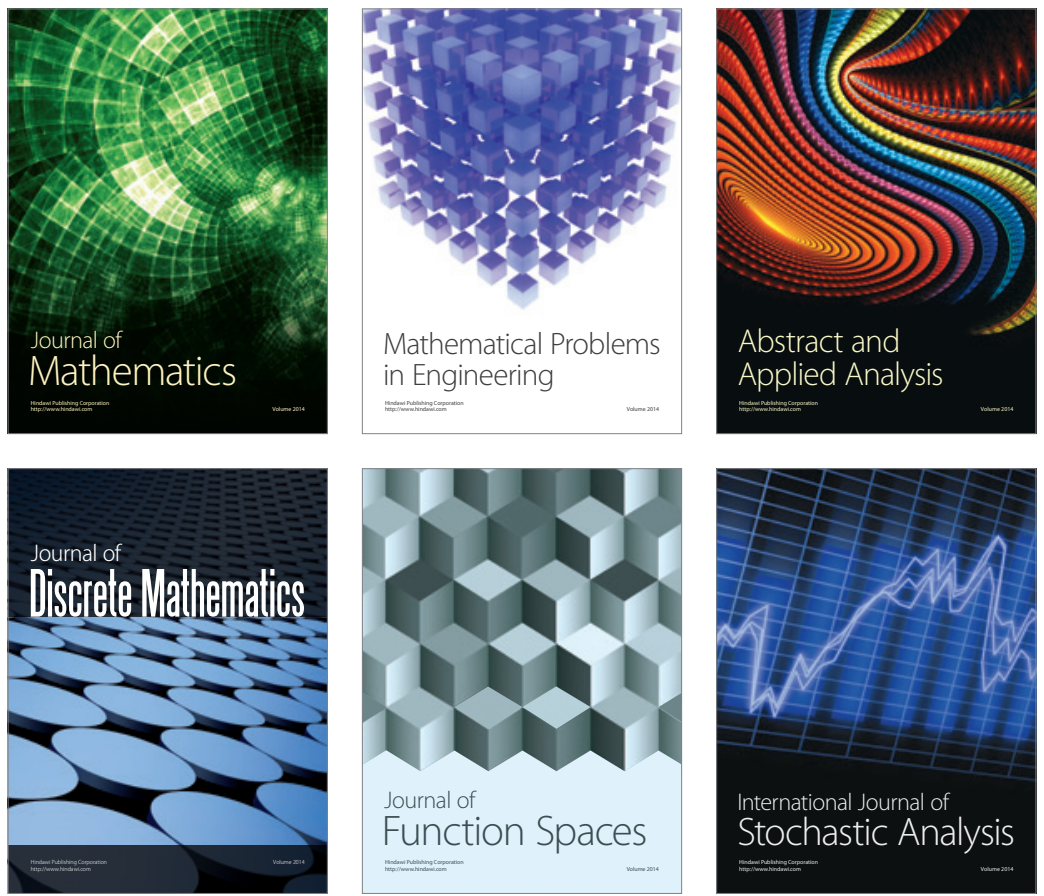

Journal of

Function Spaces

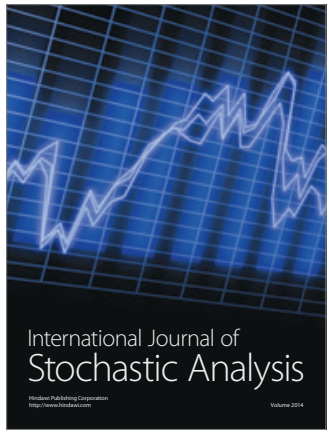

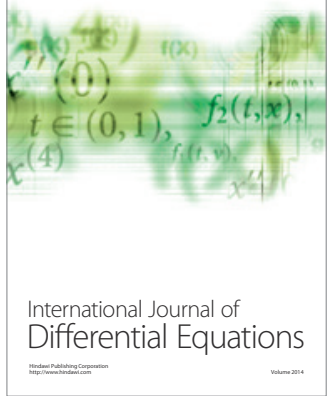
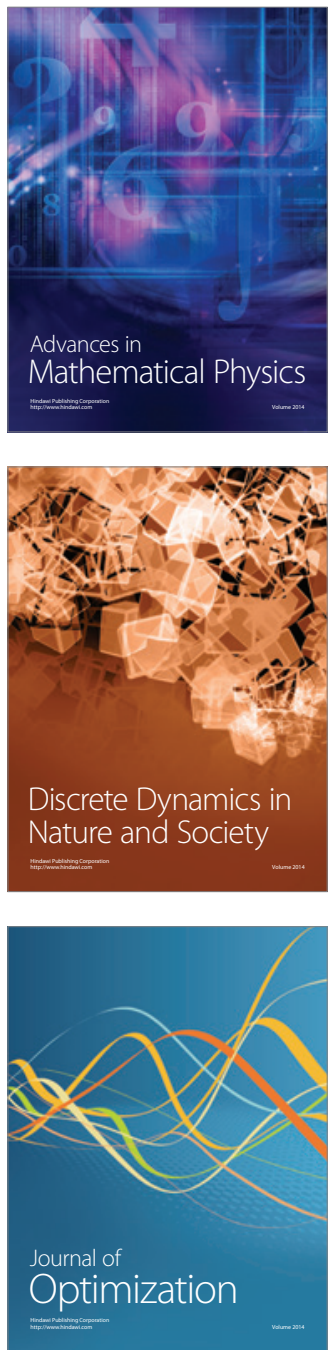